\title{
Environmental-Friendly Synthesis of Au-Te
}

\section{Clustered Nanoworms via Galvanic Replacement for} Wavelength-Selective Combination Cancer Therapy

Seounghun Kang ${ }^{2} \neq$, Yeong-Gyu Gill $\neq$, Young-Jin Kim ${ }^{3}$, Young-Kwan Kim ${ }^{3 *}$, Dal-Hee Min $^{2,4,5 *}$ and Hongje Jang ${ }^{1 *}$

${ }^{1}$ Department of Chemistry, Kwangwoon University, 20 Gwangwoon-ro, Nowon-gu, Seoul 01897, Republic of Korea

${ }^{2}$ Department of Chemistry, Seoul National University, Seoul 08826, Republic of Korea

${ }^{3}$ Carbon Composite Materials Research Center, Institute of Advanced Composite Materials, Korea Institute of Science and Technology, San 101, Eunha-ri, Bongdong-eup, Wanju-gun, Jeollabuk-do 565-905, Republic of Korea

${ }^{4}$ Center for RNA Research, Institute for Basic Science (IBS), Seoul National University, Seoul 08826, Republic of Korea

${ }^{5}$ Institute of Biotherapeutics Convergence Technology, Lemonex Inc., Seoul 08826, Republic of Korea 
"Whom to correspondence:

*Young-Kwan Kim (kimyk@dongkuk.edu)

*Dal-Hee Min (dalheemin@snu.ac.kr)

*Hongje Jang (hjang@kw.ac.kr) 


\section{Supporting data.}

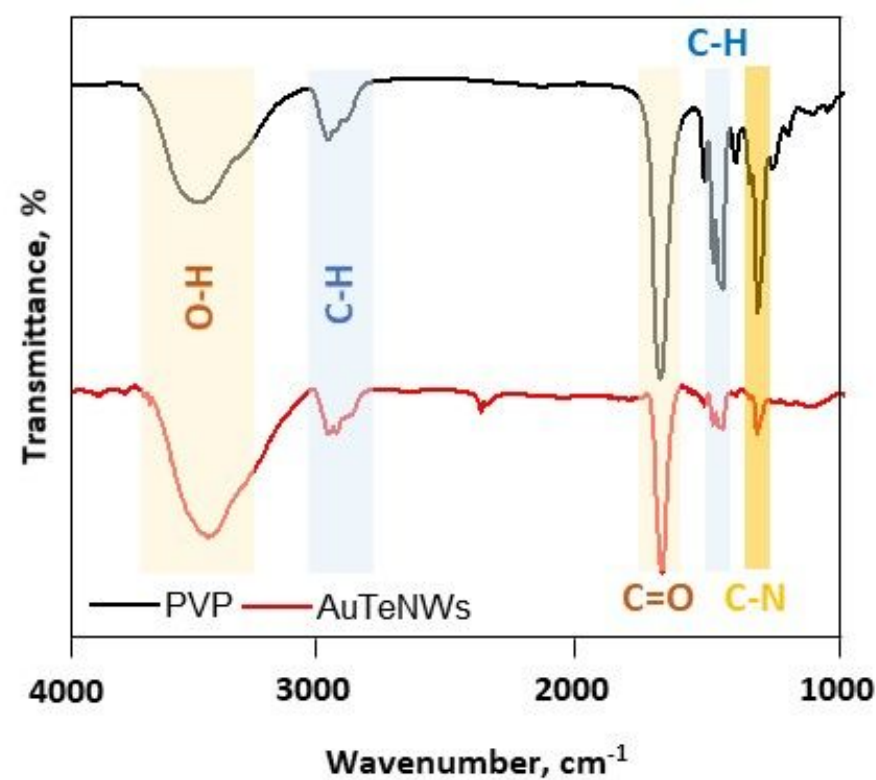

Figure S1. FT-IR spectra of free PVP (black) and AuTeNWs (red). The existence of PVP on the surface of AuTeNWs was clearly confirmed by peak assign. 
(a)

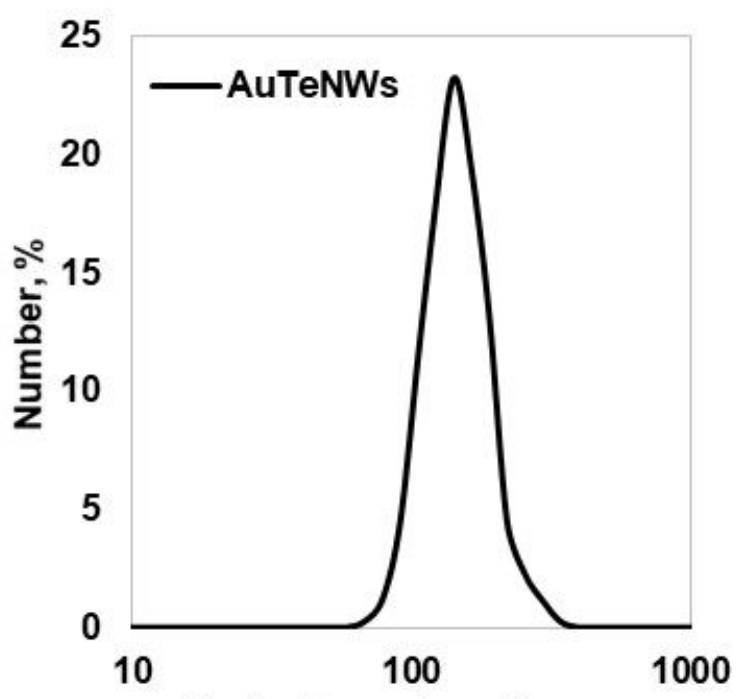

(b)

Hydrodynamic radius, $\mathrm{nm}$

\begin{tabular}{|c|c|}
\hline & Au Te NWs \\
\hline Diameter $(\mathrm{nm})$ & $164.2 . \pm 7.8$ \\
\hline Z-potential $(\mathrm{mV})$ & $-25.8 \pm 0.3$ \\
\hline Polydispersive index & $0.119 \pm 0.005$ \\
\hline
\end{tabular}

Figure S2. Characterization of AuTeNWs. (a) DLS and (b) zeta-potential measurement of AuTeNWs.

(a)

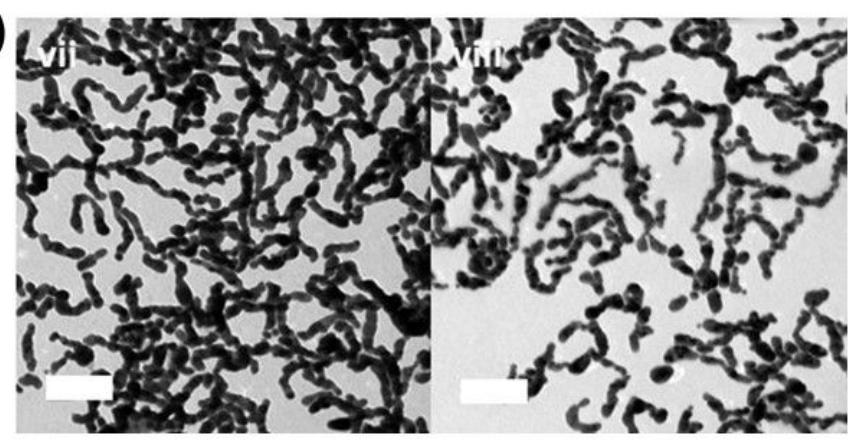

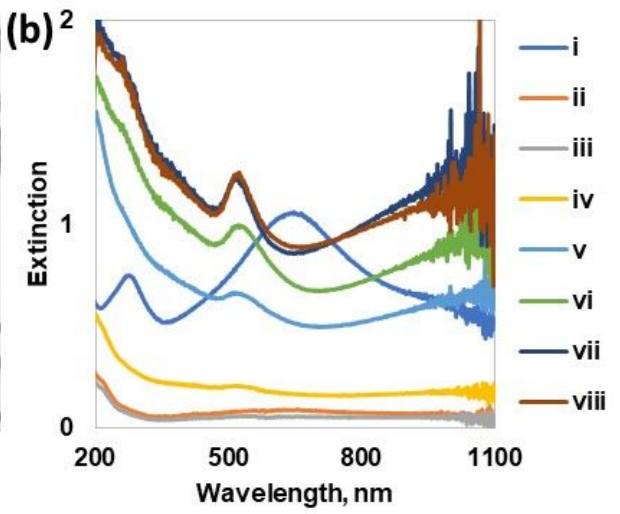

Figure S3. Synthesized AuTeNWs in the excess addition of Au(III) to TeNR templates. (a) TEM images of $X A u / T e=3.750$ (vii) and 7.500 (viii), and (b) UV-Vis-NIR spectra. The reaction was terminated to form thick nanoworm structures. According to the UV-Vis-NIR spectra, gold nanoparticle substructure peak at $525 \mathrm{~nm}$ and NIR absorption from worm-like structure was clearly observed. The scale bar is 50 
$\mathrm{nm}$.
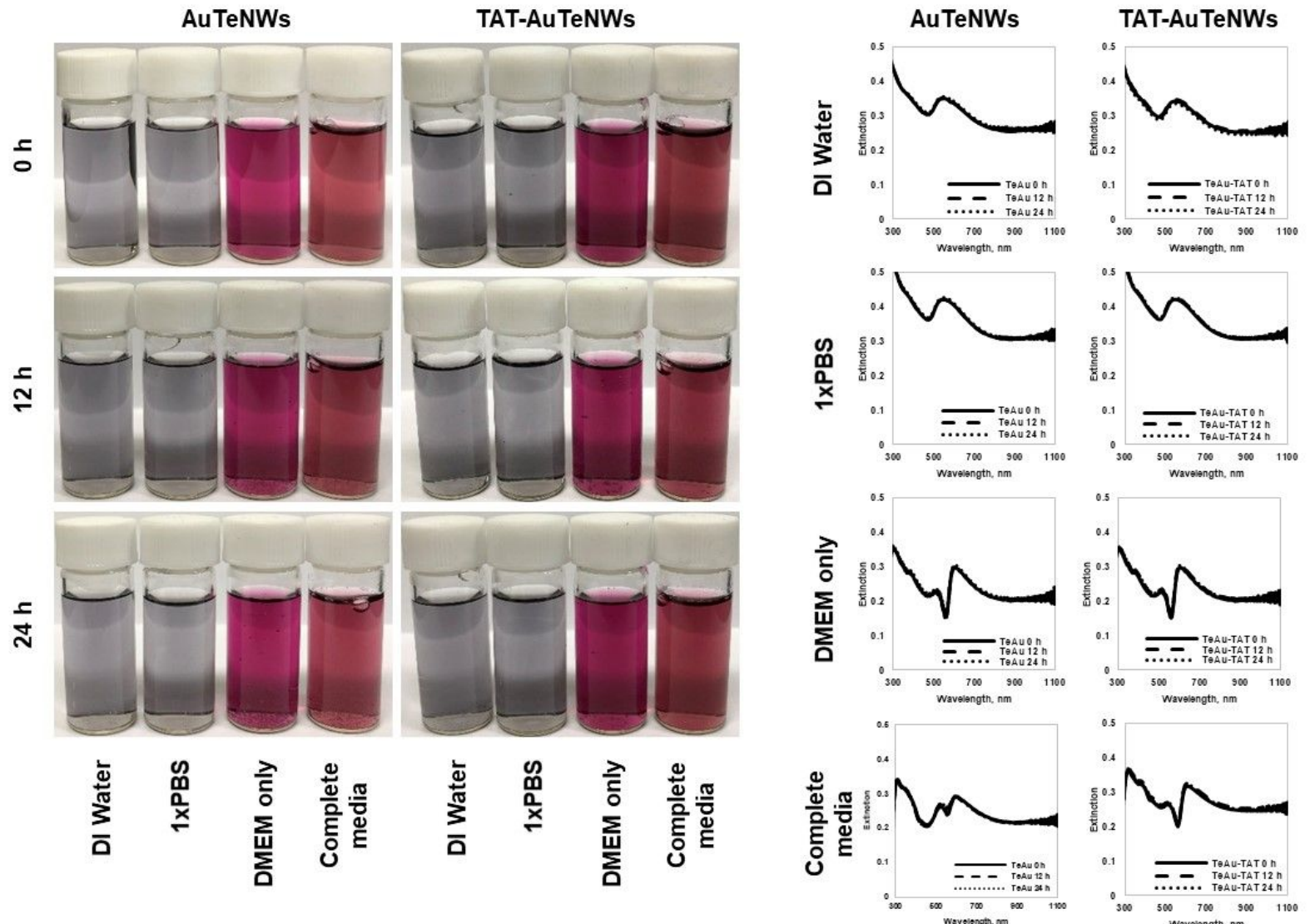

Figure S4. Colloidal stability assay of AuTeNWs and TAT-AuTeNWs. In all tested media including DI water, 1X PBS, DMEM only and complete cell culture media, both NWs did not exhibited any colloidal stability decrease. The well-maintained dispersion of AuTeNW derivatives were also observed by digital photo image. 

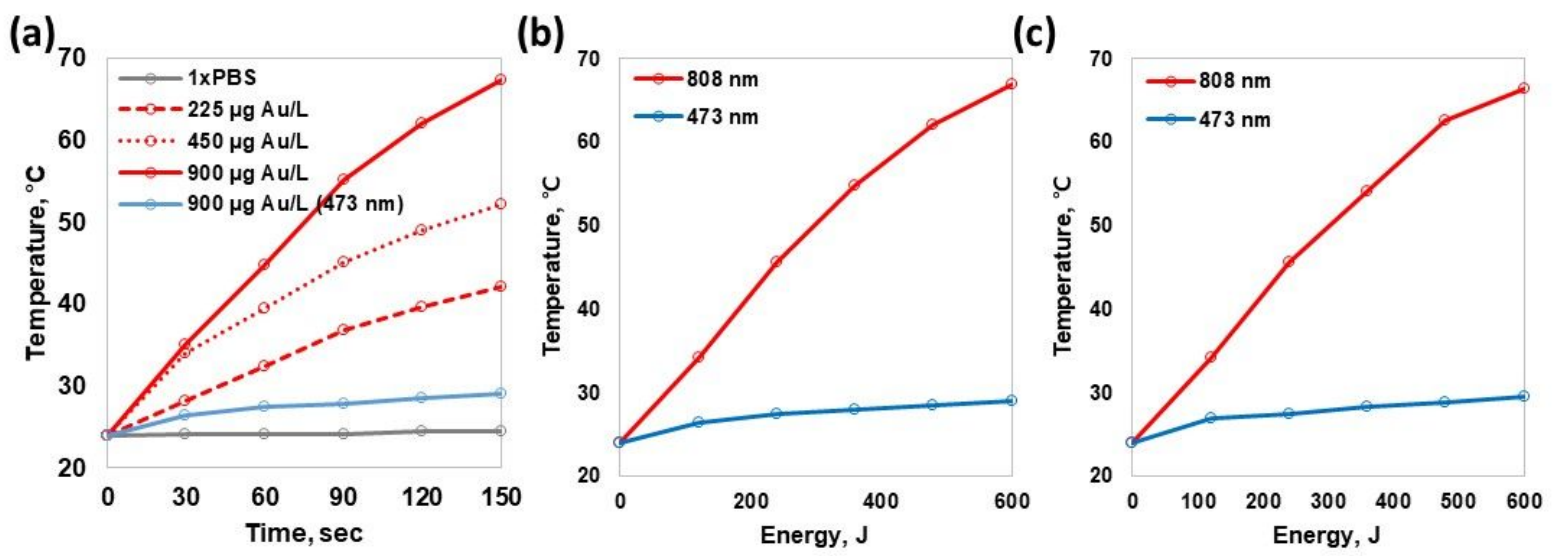

Figure S5. Temperature elevation of (a) AuTeNWs with varying the concentrations and (b) laser wavelength. (c) TAT-AuTeNWs also exhibited excellent photothermal conversion efficiency under the NIR irradiation compared with Vis.

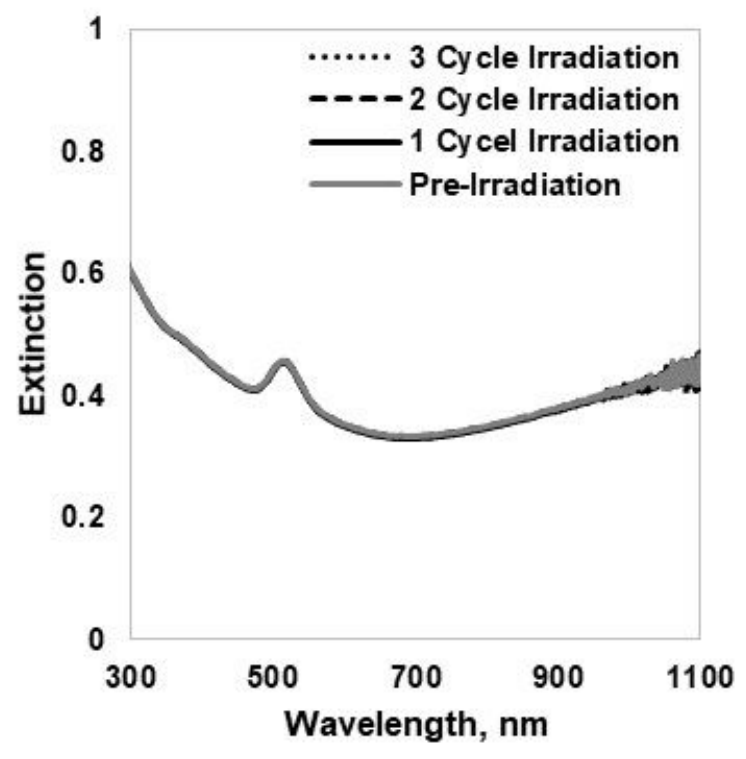

Figure S6. Photothermal durability of AuTeNWs against the irradiation of $808 \mathrm{~nm}$ diode laser. Plasmonic extinction spectra were perfectly maintained for repeated photothermal heat generation. 

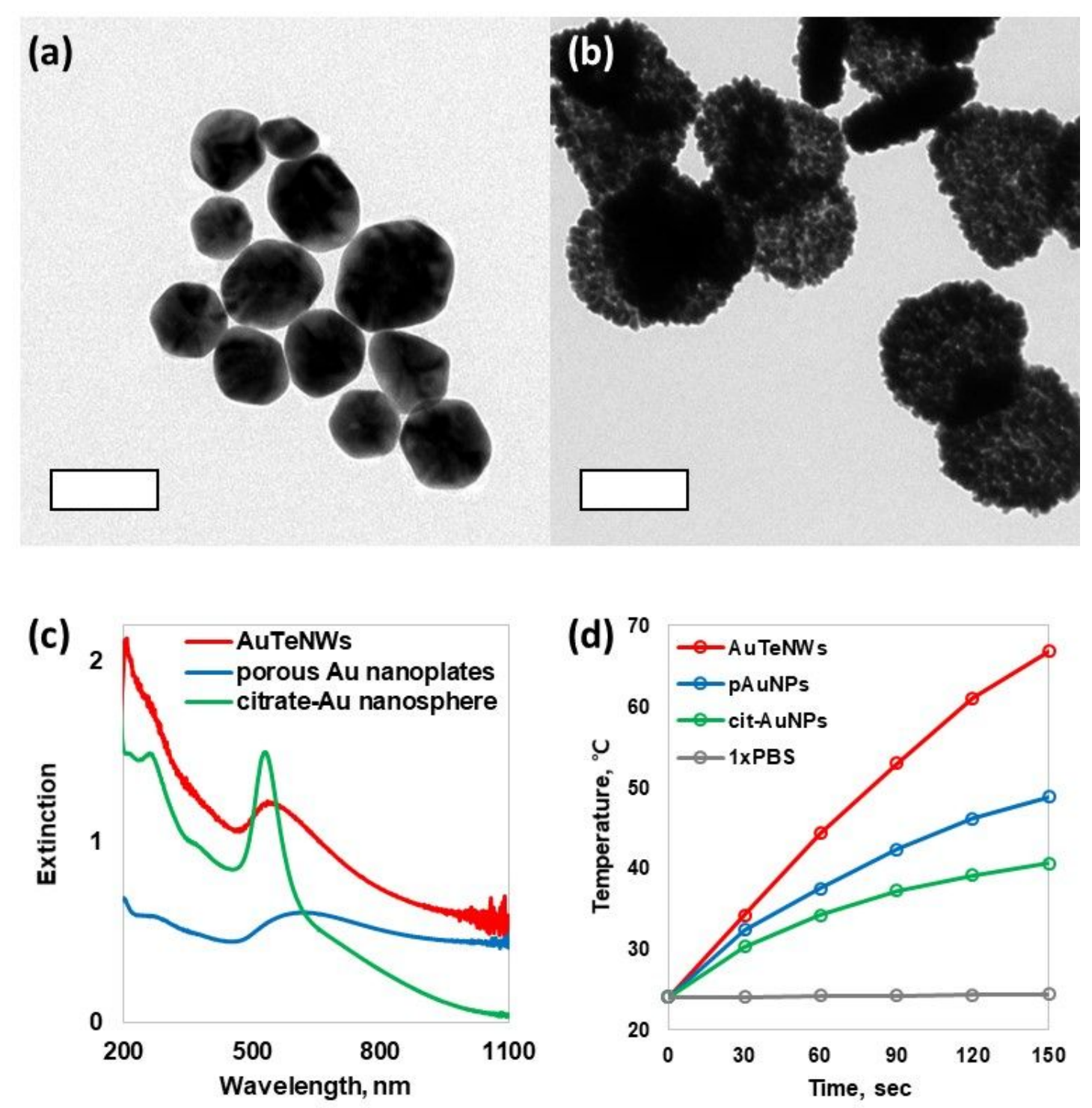

Figure S7. Gold nanoparticle derivatives for the comparison of photothermal conversion efficiency. TEM images of (a) Citrate stabilized Au nanospheres and (b) porous Au-Ag nanoplates and their (c) UV-Vis spectra with AuTeNWs. (d) Temperature elevation against $808 \mathrm{~nm}$ NIR laser irradiation. The scale bar is $50 \mathrm{~nm}$. 


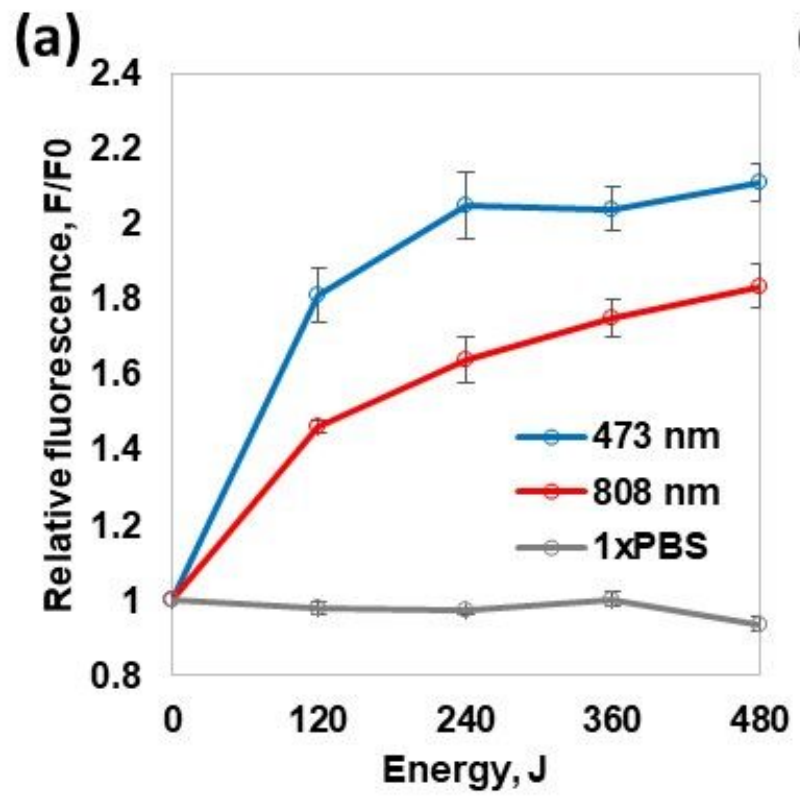

(b)

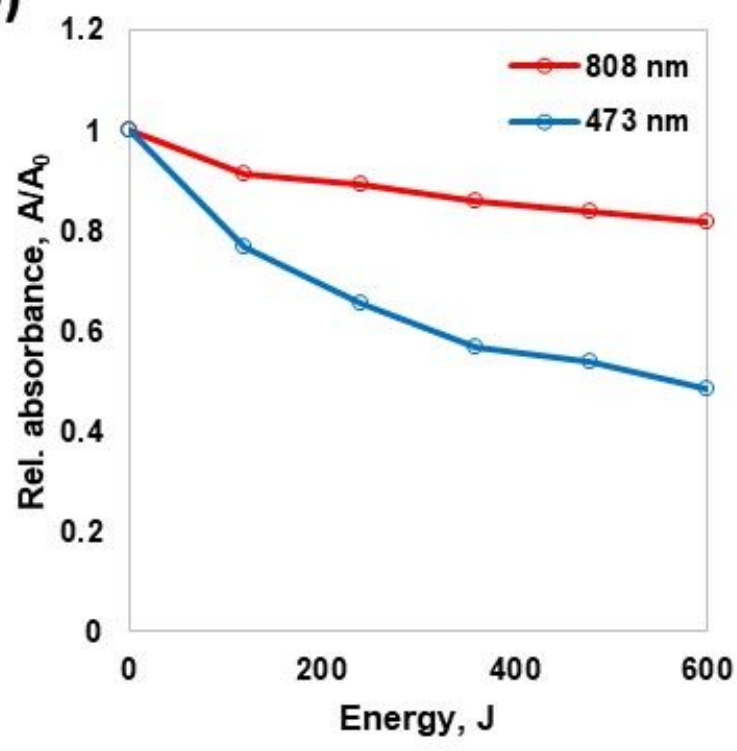

Figure S8. (a) Singlet oxygen sensor green fluorescence increase and (b) methylene blue photodegradation under the irradiation of Vis and NIR with TAT-AuTeNWs
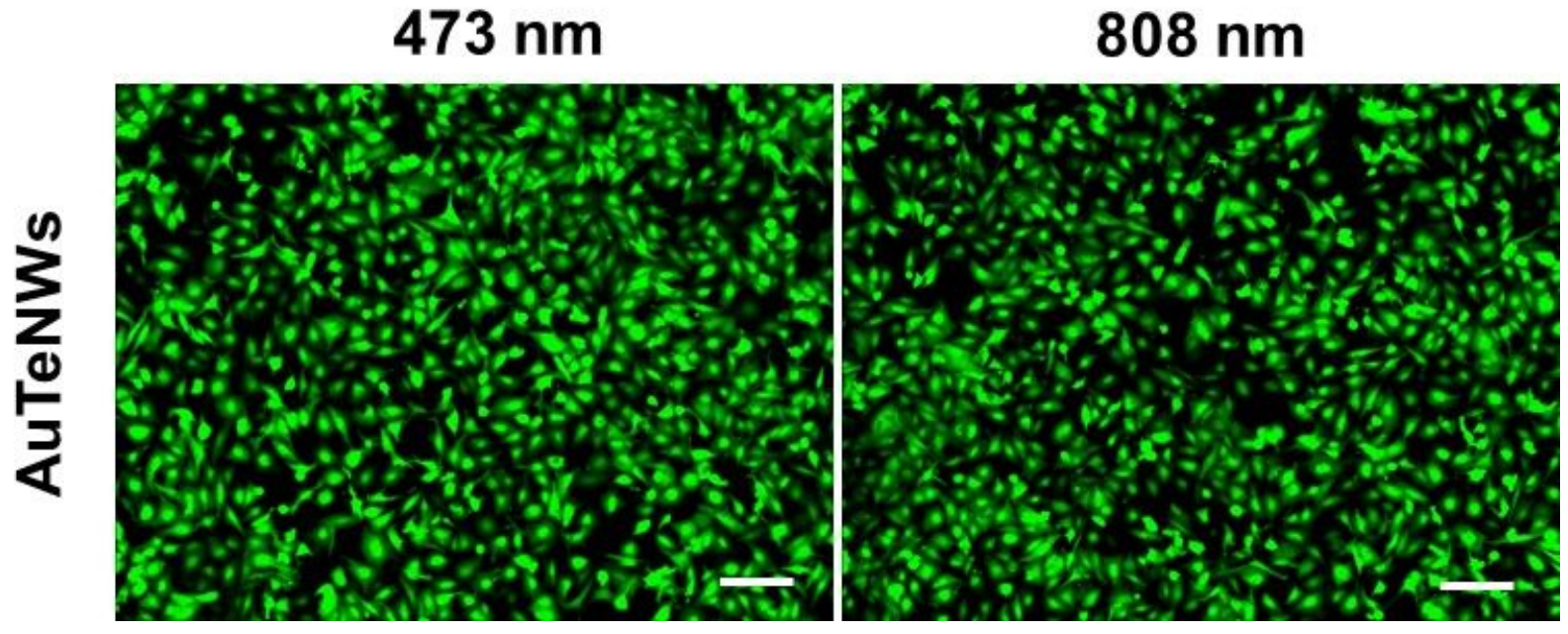

Figure S9. Live/dead stained fluorescent microscope image of 473 and $808 \mathrm{~nm}$ laser irradiation against AuTeNWs for NS3 Huh7 cells. Without the cell penetrating peptide modification, no cancer cell ablation was induced from both PDT and PTT treatment. The scale bar is $250 \mu \mathrm{m}$. 


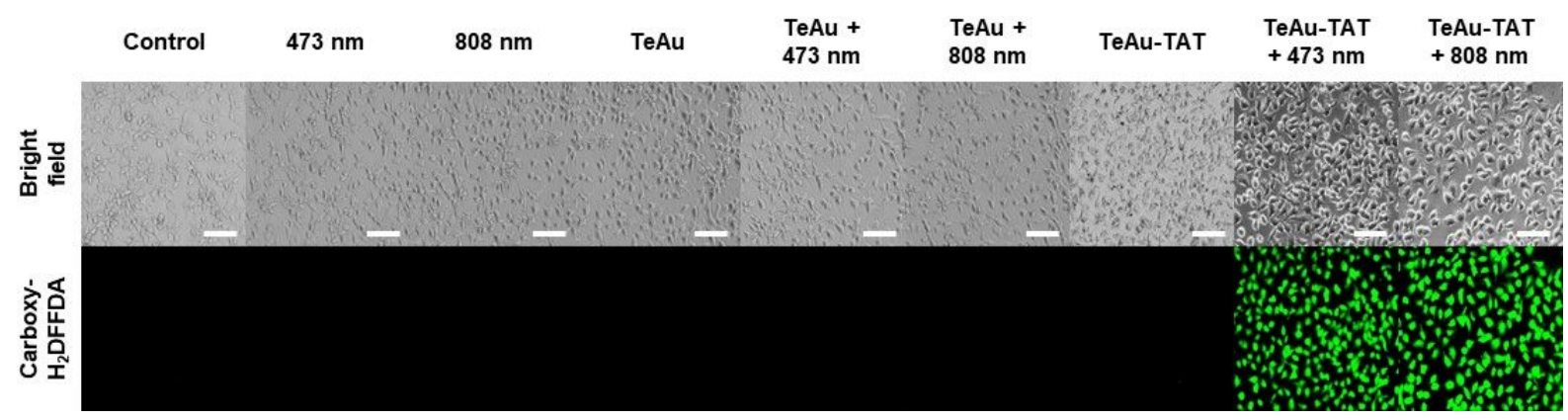

Figure S10. In vitro reactive oxygen species generation test against NS3 Huh7 cells with 473 and 808 nm laser irradiation. The scale bar is $100 \mu \mathrm{m}$. 
(a)

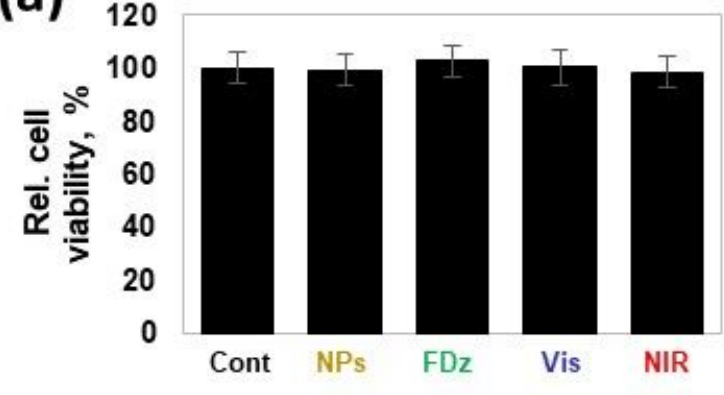

(b)

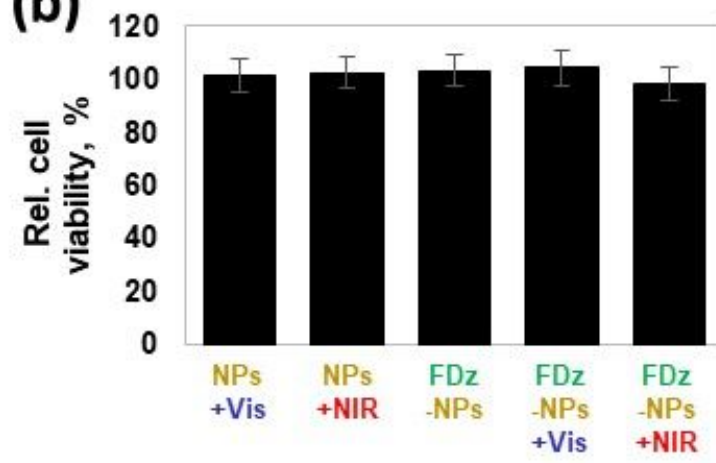

(c)

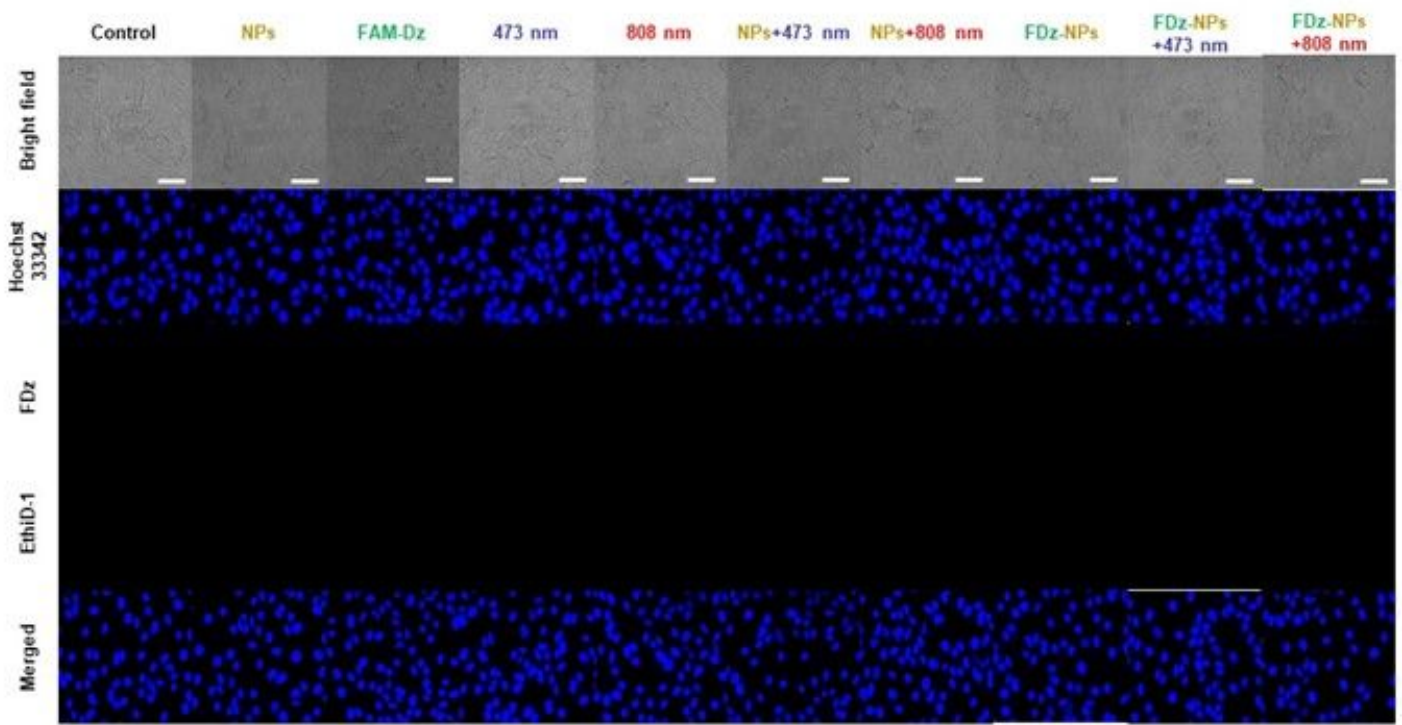

Figure S11. Control experiments for therapeutic efficacy test. (a) Control experiment conditions of untreated (cont), AuTeNWs (NPs), free FAM-Dz (FDz), $473 \mathrm{~nm}$ irradiation (Vis), and $808 \mathrm{~nm}$ irradiation (NIR) represented negligible viability decrease. (b) Mono- and dual-modal treatment of NS3 Huh7 cells with AuTeNWs also exhibited negligible therapeutic effict without cell penetrating peptide modification. (c) Fluorescence microscopy images with Hoechst 33342 (nuclei) and EthiD-1 (dead cells) staining. The scale bar is $50 \mu \mathrm{m}$. 


$$
2 \mathrm{~h}
$$

$6 \mathrm{~h}$

$12 \mathrm{~h}$

$24 \mathrm{~h}$

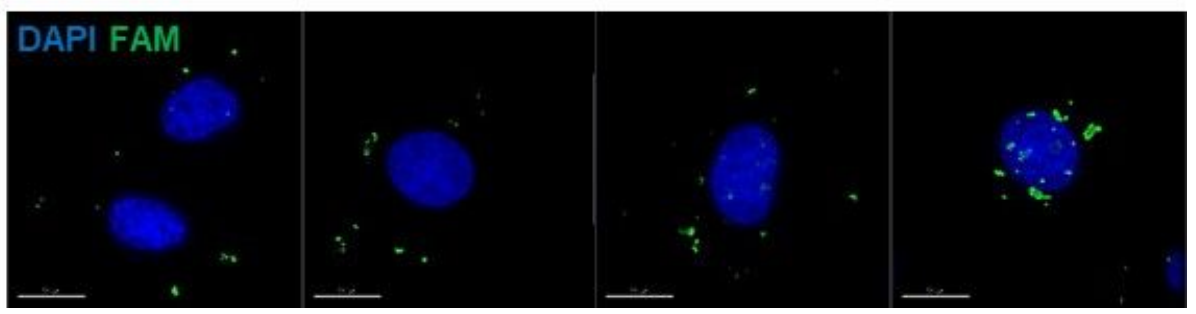

Figure S12. Intracellular distribution of FAM-Dz-TAT-AuTeNWs. Along with the incubation time, FAM$\mathrm{Dz}$ located in the perinuclear region. The scale bar is $15 \mu \mathrm{m}$.

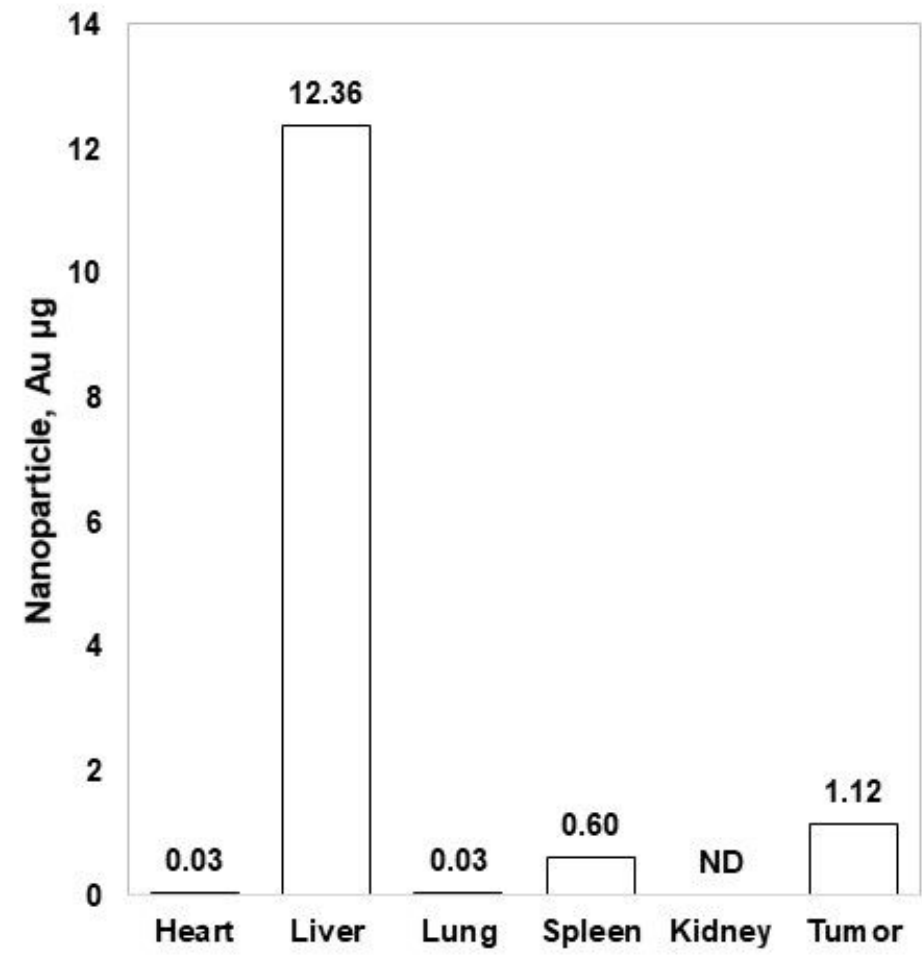

Figure S13. Mouse organ ICP-MS data. 
(a)

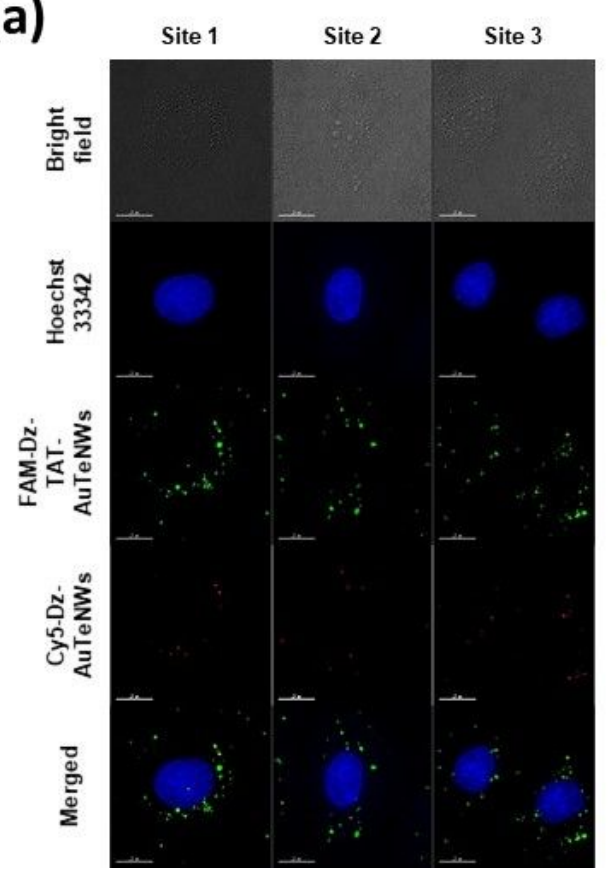

(b)

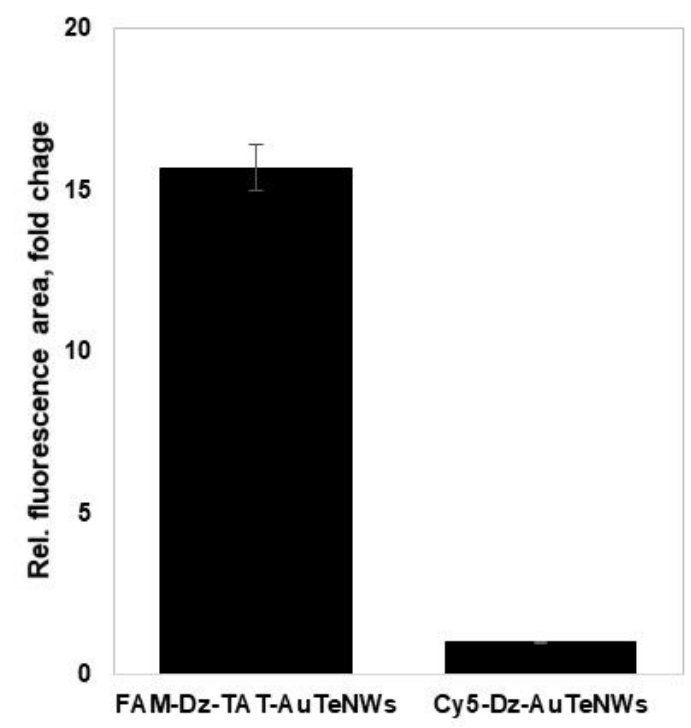

Figure S14. Cellular uptake and distribution of FAM-Dz-TAT-AuTeNWs and Cy5-Dz-AuTeNWs. The existence of TAT peptide conjugation contributed to the internalization of AuTeNWs. The scale bar is $15 \mu \mathrm{m}$. 